\title{
Light-Waves as Units of Length
}

\section{By Dr. W. Ewart Williams, King's College, London}

$\mathrm{T}$ $\mathrm{HE}$ standard of length measurement in any age gives us a fairly true picture of the general requirements of the time. King David of Scotland (c. A.D. 1150) ordained that the Scotch inch should be the mean measure of the thumbs of three men, "an merkle man, an man of measurable stature and an lyttel man", the thumbs being measured at the root of the nail. The history of the transition to our present-day standards was admirably related by Sir Richard Glazebrook in his Guthrie lecture to the Physical Society in 1931.

For more than a century, scientific men have been attracted to the idea of establishing the unit of length on the basis of some natural standard, as in the famous dream of Arago", "une mesure susceptible d'être reproduite quand même des tremblements de terre, des cataclysmes épouvantables viendraient à bouleverser notre planète et à détruire les étalons prototypes gardés aux Archives". The metre was originally intended to be one millionth part of a meridional quadrant of the earth, and the yard, if ever lost, was, according to the Weights and Measures Act of 1824, to be replaced by reference to the length of a pendulum (in vacuum) beating seconds at sea-level in London. Various difficulties finally led to the adoption of purely arbitrary units, and the metre and yard became the distances between marks on bars kept at Sèvres in France and at the Standards Office of the Board of Trade in London.

The idea of employing a light-wave as the standard of length was frequently proposed during the nineteenth century. Clerk Maxwell ${ }^{2}$, in an eloquent address to the British Association at Bradford in 1873, pointed out that: "Each molecule therefore throughout the universe bears im. pressed on it the stamp of a metric system as distinctly as does the metre of the Archives at Paris or the double royal cubit of the temple at Karnac". Pierce $^{3}$ in 1879 attempted a precision measurement of the wave-lengths of the sodium lines, and with improved diffraction gratings the work was repeated by Bell ${ }^{4}$ in 1887 . The first description of a method that would ensure sufficient accuracy was given by Michelson and Morley ${ }^{3}$ in 1887 and in more detail in 1889.

With the assistance of Dr. Gould, the American representative on the newly-formed International Committee of Weights and Measures, Michelson ${ }^{\circ}$ was able to use his new type of interferometer, and in collaboration with Benoit, succeeded in making a direct determination of the number of waves of the red radiation of cadmium con- tained in the metre. In the usual Michelson interferometer, the light first falls on a half-silvered mirror so that the reflected and transmitted beams, of approximately equal intensity, are mutually perpendicular. The beams are then reflected back on their own paths by two fully silvered mirrors. With equality of optical paths and a slight tilt of either mirror, white light fringes can be observed in the beam emerging from the semi-silvered plate.

For the purpose of the metre determination, one of the fully silvered mirrors was replaced by an 'étalon' consisting of two parallel mirrors, one above the other in a stair formation; nine such units, with mirror separations ranging from $0 \cdot 39$ $\mathrm{mm}$. to $10 \mathrm{~cm}$., were constructed so that the labour and uncertainty of counting a large number of fringes might be avoided. The exact details need not be entered into here, but it should be clearly emphasised that although only the number of fringes in the smallest unit had to be actually counted, the method was such that the number of wave-lengths in the (double) path of the largest étalon was obtained to within a few hundredths part of one fringe. A mark on this étalon was placed in line with one of the fiduciary marks of the working standard metre by means of a travelling microscope, and white light fringes were obtained in the upper étalon mirror by suitably adjusting the reference mirror of the interferometer. When the unit was moved until the fringes appeared in its lower mirror, it meant that the étalon had been displaced by exactly its own length; this procedure was repeated, in all ten times, so that any error in the determination of this basic étalon is increased tenfold. Finally, the small difference between the alignment of the second metre mark and the new position of the étalon mark is measured by means of a travelling micrometer. This distance, of the order of a few hundredths of a millimetre, is so small that any uncertainty in the previously known value of the wave-length can have no influence on the result. It should be mentioned that the working standard metre has also to be compared with the available national standards, and these in turn have been similarly compared with the prototype metre itself by means of travelling microscopes in an arrangement that is termed a line comparator.

The fringes given by a Michelson interferometer are invariably broad, however monochromatic the source may be ; in effect, we have two virtual sources, so that the bright and dark portions have equal width. 
$\mathrm{Hamy}^{7}$ in 1897 discovered the important fact that multiple reflection made it possible to obtain considerably narrower fringes with a corresponding increase in the resolving power. $\mathrm{He}$ constructed an interferometer consisting of a thinly silvered plate mounted in front of, and parallel to, a fully silvered mirror. When the fringes are observed by transmission through two semi-silvered surfaces instead of by reflection, the arrangement constitutes the Fabry-Perot interferometer, which has found wide application in the study of the structure of spectral lines.

Benoit, Fabry and Perot ${ }^{8}$ used the latter instrument as the basis of an entirely distinct method of standardising the metre in terms of wavelengths. The order of interference of the circular fringe system of an étalon with a plate separation of approximately $6.25 \mathrm{~cm}$. was first determined by means of Benoit's fractional part method, since the wave-lengths of a number of lines were already known with sufficient accuracy for this purpose. This order of interference is the number of waves contained in the double path of the étalon.

The next step was to obtain the path differenee (in wave-lengths) between a single reflection in a similar unit of twice its size, and a double reflection in the basic unit the value of which is already known. This was accomplished by arranging the units in line, so that a beam of white light could be passed through both and the well-known Brewster fringes obtained. The exact difference was found by introducing a previously calibrated wedge into the beam. The process was repeated with successively larger units until the $100 \mathrm{~cm}$. standard had been reached.

Instead of attempting to set the cross-wires of the microscope comparator on the silvered edges of the plates when comparing it with the working metre, the measurements were made to rulings on the edges of the plates close to the surface and similar in character to the marks on the metre. By means of a subsidiary experiment, probably the most ingenious and beautiful example of fine experimental work in the whole field of metrology, the correction factor, which is the total distance from these marks to the effective reflecting planes, was determined with the greatest possible precision.

The now classical work of Benoit and his collaborators, completed in 1907, was generally considered to be so accurate that a further determination was felt to be unnecessary. The wavelength of the red radiation of cadmium then found has been accepted and still remains the spectroscopic standard of length of the scientific world.

As a step towards the eventual adoption of a wave-length as the fundamental unit of length, the International Committee of Weights and Measures ${ }^{9}$ urged the various national laboratories to carry out similar investigations using their own national standards.

The first results were those of Watanabe and Imaizumi ${ }^{10}$. Their apparatus, constructed by Hilger, was almost identical with that of Benoit, Fabry and Perot. A difference of 0.002 A. was initially observed between the values of the wavelength of the red line as determined from the two Japanese standards No. 10 and No. 20. Agreement was obtained when these prototypes were later compared with the International substandards at Sèvres, and fresh values were found for their exact individual length.

The results of a determination carried out at the Physikalische Technische Reichanstalt, Berlin, have been given by Kösters ${ }^{11}$. The details of the method have not yet been given, but presumably, in view of the earlier intercomparisons of the cadmium and krypton wave-lengths described by Weber and Lampe ${ }^{12}$, the method is based on Kösters's application of the Twyman and Green ${ }^{13}$ interferometer. This consists of a modification of the Michelson instrument, in which the straight localised fringes are observed by using a point source at the focus of a lens, the eye replacing the eyepiece of the telescope.

Sears and Barrell ${ }^{14}$, of the Metrological Department of the National Physical Laboratory, have recently published the preliminary results of a systematic investigation of the problem extending over the last ten years. A description of the actual apparatus has been previously given ${ }^{15}$. As will be seen in a later section in which the various results are analysed, the work is of the greatest importance; and not only the authors but also the Laboratory and its directorate deserve to be congratulated on the initiation and planning of such a comprehensive programme, the first stage of which has been completed.

The authors have approached the problem from an entirely different angle; instead of setting themselves the problem of finding the number of waves contained in the metre, they have primarily determined the number contained in standard gauges of approximately one metre length. This they can find with an accuracy far exceeding that possible in a direct metre determination, so that the lengths of various other gauges (in terms of wave-lengths) can be ascertained with a precision hitherto considered impossible.

The first part of the method is basically that of Benoit, Fabry and Perot. The number of waves in a $\frac{1}{12}$ or a $\frac{1}{9}$ metre étalon is determined and the unit is used for direct comparison with a $\frac{1}{3}$ metre étalon by means of Brewster's fringes. The step-up of four or three times instead of the 
double step formerly used only involves a slight reduction in the clearness of the fringes. The calibrated wedge is omitted and the exact multiple of optical paths is obtained by tilting the larger unit, a method due to Fabry and Buisson ${ }^{16}$.

An $\mathrm{X}$-section gauge, the ends of which are optically parallel, is mounted inside the third étalon, which has to be slightly longer than a metre in order to accommodate the metre gauge. The Brewster fringes, given by three reflections in the intermediate standard and one in the larger unit, are observed in turn through each of the four channels or apertures formed by the Xsection. Thus the mean length $A$ of the largest étalon in wave-length units becomes known. The ends of the $\mathrm{X}$-gauge do not touch the half-silvered étalon surfaces; the path differences $B$ and $C$ between the polished ends of the gauge and the silvered surfaces are determined by means of the circular Hamy fringes using Benoit's fractional part method. The optical length of the gauge is therefore $A-B-C$ wave-lengths, and its mechanical length is also known provided the phase change on reflection at the gauge surfaces is determined by a subsidiary experiment. This has been the primary purpose of the investigation.

In order to obtain the number of waves in the metre, an auxiliary gauge approximately half an inch shorter is employed, together with two further half-inch end gauges at the centres of which fine lines are engraved. If a half-inch gauge is wrung in contact with the auxiliary, a metre end standard is obtained which can be compared directly by interferometric methods with the $\mathrm{X}$-section gauge. When both blocks are wrung, one on each end of the auxiliary, the composite gauge forms a metre line standard which can be compared with the national standard in a line comparator. By taking observations with the blocks contacted in all possible ways, the effects of slight irregularities and differences in the blocks and their bisecting lines are eliminated.

In the determination of the yard, the same étalons were used but the $\mathrm{X}$ - and the auxiliary gauges were 36 and 35.5 in. respectively, so that the differences $B$ and $C$ were therefore much greater.

Reference should also be made to the determination of the yard by Tutton ${ }^{17}$. The method involved the actual counting of the number of fringes in a distance of $\frac{1}{16}$ in. By an ingenious system of multiplication, the number of wavelengths in the yard is determined by means of the Tutton wave-length comparator. The basic unit is, however, too short to yield sufficiently accurate results. This can be seen from the fact that temperature and pressure corrections for the basic unit are negligible, while they are applied to the greater lengths determined in terms of this unit. On the other hand, the accuracy is considerably greater than the one part in fifty thousand which a casual inspection of the method seems to imply.

For the sake of completeness, it might be recorded here that the writer ${ }^{18}$ has proposed an entirely different method whereby the length of a metre gauge in the wave-lengths may be directly obtained from two observations. A suitable reflection echelon is to be used and any errors due to optical multiplication are eliminated.

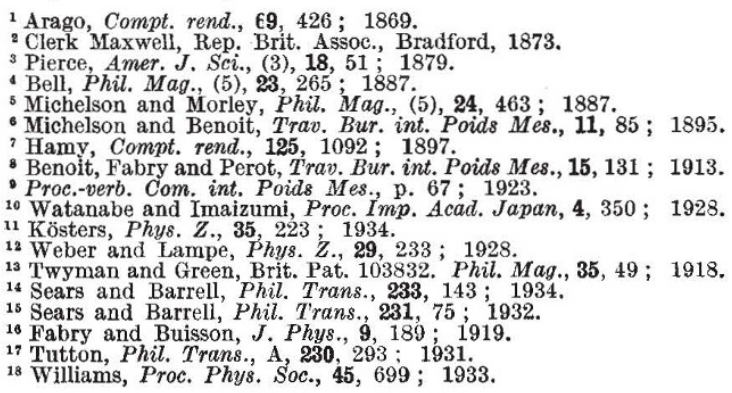
(To be continued.)

\section{Obituary}

Prof. M. I. Pupin

$\mathrm{B}^{\mathrm{Y}}$ the death of Michael Idvorsky Pupin in New York on March 12 at the age of seventy-six years, we lose a mathematical physicist who has played an important part in engineering progress since the end of last century. The son of Serbian peasants, he emigrated at the age of sixteen years to New York, where he started to earn his living with only five cents in his pocket. After many struggles, during which he supported himself by manual labour, he gained free tuition at the entrance examination to Columbia University in 1879 and distinguished him. self by winning many prizes both for his studies and at athletics. After graduating, he became a naturalised citizen of the United States.
Pupin was the first holder of the John Tyndall fellowship at Columbia. He elected to complete his training at Cambridge, England, being attracted by the prospect of studying under Clerk Maxwell and learning about his electromagnetic theory of light. Marion Crawford, the novelist, gave him a letter of introduction to Oscar Browning of King's, but he was away on his summer vacation. Pupin also had a letter of introduction to W. D. Niven, a tutor of Trinity College, who asked him what was his object in coming to Cambridge. He replied that he wanted to study under Clerk Maxwell, and was very surprised to learn that Maxwell had been dead for four years.

Before settling down at Cambridge, Pupin went to 